Besonders schmerzlich ist, daß Strohl uns in einem Moment entrissen wurde, in welchem er eben im Begriffe stand, diese kleine naturwissenschafts-historische Zeitschrift herauszugeben, um gewissermaßen in einem Kristallisationspunkt all das zu sammeln, was an lebendigen Kräften auf diesem Gebiet in der Schweiz tätig ist. Möge es trotzdem gelingen, diesen Kristallisationspunkt, den Strohl als geistiger Mittel- und Anziehungspunkt wie kein zweiter zu bilden geschaffen war, in die Wirklichkeit umzusetzen.

Und noch ein Gedanke bewegt uns schmerzlich: wie hätte der Mann, dem Verständigung über alle nationalen Bindungen und Grenzen hinweg eine Herzensangelegenheit war und dem die Überwindung materieller und geistiger Widerstände und vorgefaßter Meinungen dank seiner schöpferisch vermittelnden Art wie selten einem gelang, im Großen als Vermittler wirken können, wenn es einmal gelten sollte, die schmerzlich zerrissene, vielfach unheilbar zerstörte materielle und geistige Welt wieder aufzubauen und jenen geistigen Consensus wieder herzustellen, ohne den sie, die zum Kosmos berufene, nicht zu leben vermag.

So ist, viel zu früh, der schweizerischen Wissenschaft eine ihrer markantesten Gestalten durch vorzeitigen Tod entrissen worden. Umso lebendiger wird Strohls Andenken bei denjenigen weiter wirken, welche freundschaftlich mit ihm verbunden, sein Werk als posthumen Auftrag mit bescheidenen Kräften weiterzuführen sich verpflichtet fühlen.

Hans Fischer.

\title{
Souvenirs sur A.-C. Klebs
}

Tant de milliers de jeunes hommes jour après jour voués à la mort, sur toute l'étendue de notre globe, ces hécatombes n'ont-elles pas émoussé notre sensibilité? Il me semble qu'elles l'aiguisent plutôt; les deuils qui nous touchent de près nous en deviennent plus sensibles. Sur le fond de l'universelle misère, ces gouttes d'amertume ne sont que plus amères. Avec tant de souffrance épandue partout, nous voudrions que ces souffrances particulières nous soient épargnées. Vœu, hélas, aussi vain que tant d'autres.

C'est ainsi que nous venons d'être frappés par la mort d'Arnold Klebs. 
Voilà un quart de siècle qu'il était devenu des nôtres, depuis qu'il s'était fixé à Nyon, dans cette maison des Terrasses, construite par une enfant de Nyon devenue duchesse par la grâce du théâtre, et qui transplanta une sorte de palazzo sur la rive du Léman. Les encadrements de fenêtres apportés de Venise n'étaient point pour choquer Klebs, qui appréciait le paysage et s'était borné à planter sur le toit un fût de cheminée savoyard emprunté à Chillon. Son esprit cosmopolite se sentait chez lui devant l'horizon d'eau, de cultures, de montagnes. On devinait dans le lointain ce château de Ripaille, où un duc de Savoie avait, il y a cinq siècles, rêvé de se retirer dans un ermitage groupant quelques sages, amis des lettres; projet qui devait sourire à notre ami mieux qu'à personne.

Tout proche, sur l'autre rive de la baie de Nyon, se détache le Prieuré de Promenthoux, dont l'histoire était faite pour toucher de plus près encore le maître des Terrasses; n'avait-il pas été, autour de 1450, le domaine de maître Gerbin Cruse, de ce médecin notable, au service de la ville de Chambéry, puis d'Amédée VIII l'ermite de Ripaille, avant de se fixer à Genève où il sera membre du Conseil des Cinquante? Et père, surtout, de Louis Cruse, l'imprimeur auquel sont dus tant d'incunables, dont l'un est sorti de presse à Promenthoux même, le 2 août 1482! A ma dernière visite, un après midi de septembre 1942, le soleil dispensait généreusement ses rayons. Une douceur pénétrante était épandue sur le pays; tout était image de paix. Pourtant, la maîtresse de la maison, frappée par la maladie, ne sentait plus rien de cette beauté; au-delà de nos frontières, guerre, ruine, asservissement, partout; l'hiver et ses bourrasques étaient à la porte. Pendant que nous regardions les voiles glisser sur l'eau unie, dans la lumière dorée, mon hôte me contait les visites de son ami Fritjof Nansen, alors que celui-ci fréquentait Genève pour la Société des Nations: ce qu'ils aimaient, c'était profiter d'un orage pour hisser la voile et courir de dangereuses bordées. Ainsi, sous l'apparente douceur d'une merveilleuse journée, le déchaînement des contrastes, ceux de la vie humaine et ceux de la nature, n'étaient que voilés. N'était-ce pas l'image même de la vie de notre ami?

Nyon n'en fut, toutefois, que la dernière phase. La première avait été consacrée à la médecine. Il y était venu tout naturellement. Son père était professeur; Berne, Prague, Zurich, avaient bénéficié de son enseignement; né à Berne, Arnold fit ses études en Suisse et prit à Bâle son grade de docteur. Edwin Klebs, chercheur aux vues personnelles, 


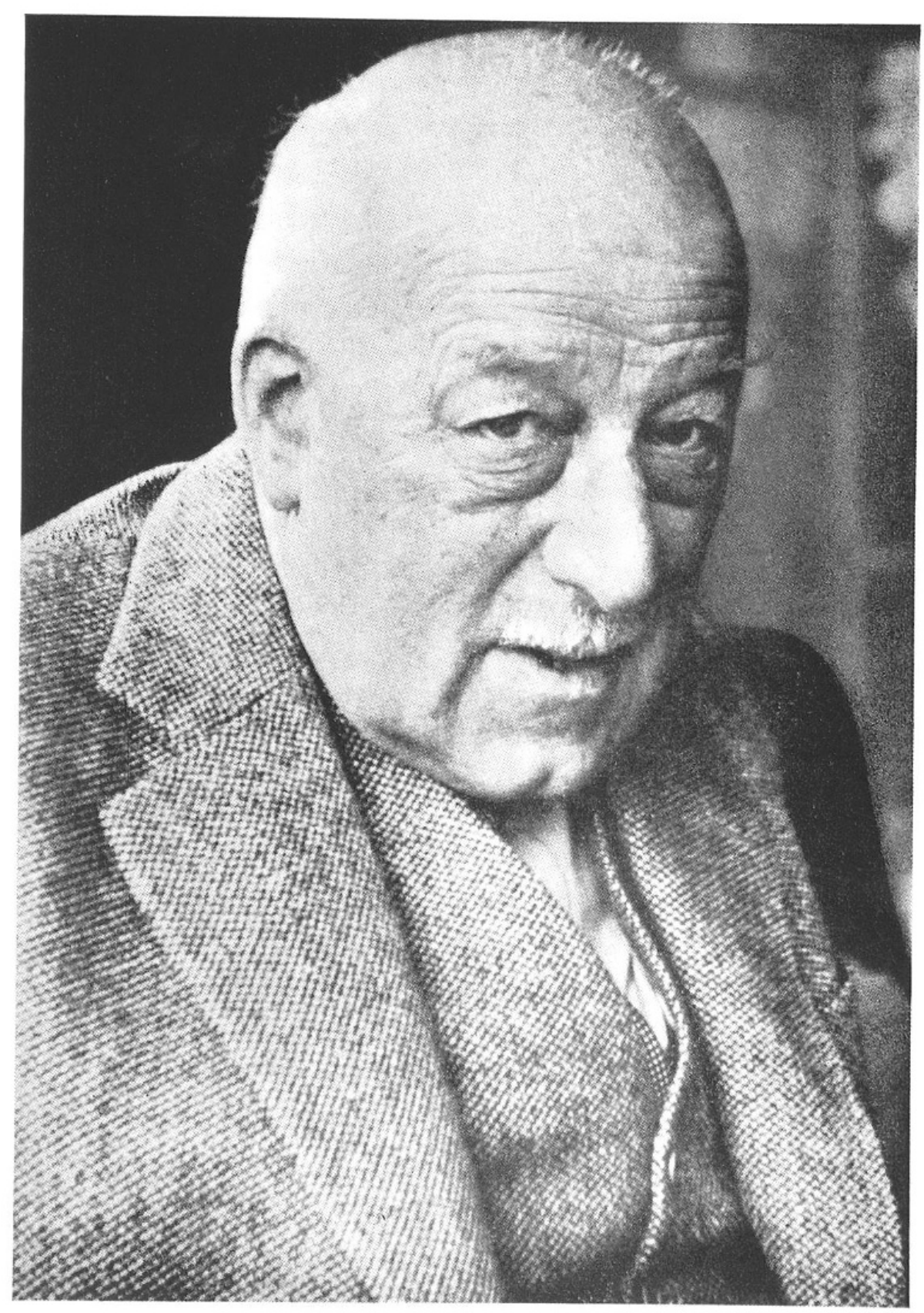

Arnold Klebs 
élève de Virchow, contemporain des Recklinghausen, des Robert Koch, avait sur bien des points ouvert des voies nouvelles, participé à des découvertes dont le renom fut parfois cueilli par d'autres. Il lui manquait un certain équilibre, la faculté de surmonter les obstacles par patience et longueur de temps, plutôt que de foncer sur eux par force et violence. Il avait du moins eu la sagesse, après s'être fait naturaliser bernois, de choisir pour femme une bernoise, authentique fille du terroir, bien faite pour apporter dans le ménage un élément de stabilité. Leur fils dut bénir souvent l'influence apaisante exercée par elle sur le trop impatient auteur de ses jours.

C'est en Amérique qu'Arnold Klebs fit sa carrière médicale. Séduit par l'intensité de la vie de ce pays jeune, par la latitude qu'il offrait à chacun de courir sa chance, il en acquit la nationalité; sans doute pensait-il y être fixé définitivement. Ses débuts furent brillants. Médecin praticien, médecin consultant, il avait vite conquis une position en vue. Consacré à l'étude de la tuberculose, il avait fondé un sanatorium dans l'Alabama, puis créé à Chicago le Tuberculosis Institute; c'est à son instigation que se constitua la National Tuberculosis Association, au comité de laquelle il appartint pendant les difficiles années du début; c'est lui encore qui fit appeler von Pirquet aux Etats-Unis. A côté de nombre de mémoires spéciaux, il avait édité un volume considérable, où sous sa direction les principaux représentants de cette discipline aux Etats-Unis avaient résumé l'état des connaissances de l'époque. Puis, brusquement, il abandonna ses premières amours. Les conclusions auxquelles il était parvenu lui enlevaient le goût de continuer sur la même voie. Il avait, estimait-il, touché le nœud du problème, et c'était là ce qui, au fond, le passionnait; l'application des mesures pratiques pouvait être laissée à d'autres. Il renonça à la pratique médicale. Ne prenons pas ce geste pour l'indice d'une inquiétante versatilité. Ce qui poussa Klebs à brûler ses premiers dieux est au contraire la volonté d'être fidèle à sa nature profonde. Il ne pouvait rien conserver dans sa vie qui ne fût pas fondé sur le roc de la conviction la plus sévèrement contrôlée.

Sur quoi survint la première guerre mondiale. Il passa une partie de ces années dans la grande bibliothèque médicale réunie à Washington par les soins du médecin de l'armée, la Surgeon General's Library; et là, sous l'impulsion de Garrison, s'ouvrit la porte de la seconde étape de sa vie, l'étude de l'histoire de l'esprit humain dans le domaine des sciences médicales. Une période retint plus que les autres son intérêt, l'âge de la 
Renaissance, où l'invention de Gutenberg vint brusquement transformer les moyens d'étude et permettre la diffusion prompte et étendue du savoir. Rares et coûteux jusque là, mis à la disposition seulement de quelques privilégiés isolés, voici qu’en quelques années les livres sont, par centaines, par milliers, accessibles à chacun. Aurore merveilleuse, œuvre du seul génie humain! Comment n'en pas augurer un avenir de bonheur? Ces incunables, ces nouveau-nés des presses, que les centres de culture mettent au jour partout, ne sont-ils pas les prémices de la plus précieuse récolte, celle de la sagesse, mise à la portée de tous? - Il a fallu l'expérience de notre temps pour montrer que du meilleur au pire il n'y a qu'un pas.

Préparé à ce nouveau labeur, ayant visité toutes les grandes bibliothèques riches en incunables, Klebs se créa dans le Cottage des Terrasses sa bibliothèque de travail. S'il connaissait comme personne les vieux livres et savait les apprécier, il n'était pas un bibliophile au sens étroit du mot, l'amateur pour lequel la rareté, la singularité d'un ouvrage, son papier ou sa reliure, en sont l'attrait principal. Il appréciait la beauté du texte ou des images, car il avait une sensibilité esthétique très fine, dessinant et peignant lui-même d'une main habile; mais ce qu'il cherchait avant tout était la place de chaque auteur dans le mouvement des idées. Quel était son apport personnel; quelles influences avait-il subies; quelles traces ses idées laisseront-elles chez les après-venant? - Voilà ce qu'il s'efforçait d'établir. Problèmes infiniment complexes, qui paraissent simples seulement à ceux qui se contentent des perspectives offertes par les manuels. Avant de prendre position, de se permettre un jugement personnel, des études minutieuses sont nécessaires, portant sur les plus infimes détails aussi bien que sur les grands courants d'idées. Ce n'est que pas à pas, par un labeur parfois rebutant et qui en devient héroïque, que certains points peuvent être fixés et finiront par servir de fondement à une construction solide.

Vingt-cinq ans de ce travail aboutirent à la publication des Incunabula scientifica et medica. Simple liste, dépouillée à l'extrême, ne donnant que le strict nécessaire permettant l'identification assurée de chaque numéro. Pour un millier d'ouvrages, chaque impression antérieure à 1501 est notée et caractérisée; et s'il en est qui n'ont été édités qu'une fois, d'autres figurent au tableau avec trente éditions ou davantage. Tout cela, distingué suivant l'imprimeur ou l'éditeur, le lieu, la date; autant de précisions que l'ouvrage ne prend pas toujours la peine de fournir et 
qu'il a fallu reconstituer à force d'ingéniosité et de rigueur, par des comparaisons et des recoupements sans fin. C'est là que le coup d'œil aigu de notre ami, la sûreté avec laquelle il saisissait les types généraux d'impression, leurs particularités régionales ou personnelles, l'amenaient à des conclusions fermes, là où d'autres n'eussent trouvé qu'occasions d'hésiter ou d'errer; à moins qu'il n'aboutît, au contraire, à des réserves, insoupçonnées par des esprits moins avertis.

L'étendue de son information, la sûreté de son jugement, sa promptitude, parfois, étaient extraordinaires. Désireux d'examiner certains incunables d'une bibliothèque publique, il fut un jour reçu par un conservateur qui poussait la méfiance au-delà des limites habituelles et pour l'un de ces trésors, en particulier, ne put se résoudre à le confier, ne fût-ce qu'un instant, aux mains de cet étranger trop curieux. Personne, disait-il, n'avait encore établi l'origine de cet exemplaire, qui semblait unique; il regrettait, mais il n'osait vraiment autoriser le visiteur à le feuilleter; le règlement, vous savez ... Et tout à ce qu'il tenait pour son devoir de Cerbère, il feuilletait lui-même cette merveille, s'attardant un instant ici, un instant là... Je vous remercie, lui dit enfin Klebs; et pour vous tranquilliser vous dirai que votre bouquin vient de tel imprimeur et est sorti en telle année de son atelier en telle ville. Avec ce qu'il connaissait des autres éditions, quelques regards jetés à la dérobée lui avaient suffi pour être fixé. L'ultime grain de sel vint plus tard encore assaisonner cette rencontre de deux types d'hommes: le bibliothécaire publia une notice où figurait la conclusion que Klebs lui avait fournie, mais donnée comme le fruit de ses propres recherches, dans un domaine jusqu'alors inexploré...

J'ai cru, jusque tout récemment, avoir un autre indice de l'extraordinaire perspicacité de Klebs dans le fait suivant qui concerne les Cruse, père et fils, mentionnés plus haut. La présence du père à Promenthoux me faisait chercher des précisions sur ce maître ès arts et en médecine, et je n'en trouvais point en dehors de Genève et des archives vaudoises, où aucune pièce ne donne son origine. Klebs me l'indiqua aussitôt: il était d'Attendorn au diocèse de Cologne et devait être né vers 1399 car 1413 le voit immatriculé à l'Université et il y prend son premier grade en 1415. Si Klebs avait dirigé ses investigations de ce côté, c'est que certaines particularités des livres de Louis Cruse lui avaient fait pressentir une influence de traditions de Cologne... Aujourd'hui, pourtant, où il n'est plus là pour me permettre de fixer

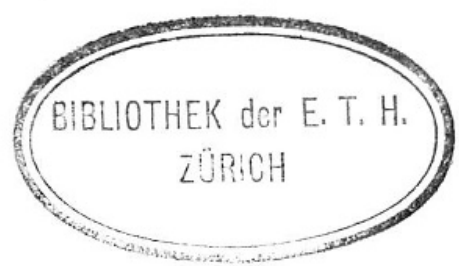


ce point, je ne suis plus aussi certain que cette sorte de divination ait bien eu la valeur que je lui attribuais. J'ai en effet constaté récemment, par des documents savoyards, que lors de sa nomination comme médecin de la ville de Chambéry (1426-39), maître Gerbin Cruse se donne pour originaire de Cologne. Klebs en avait-il connaissance, je ne le crois pas; mais ce n'est pas absolument impossible.

Il eut, heureusement, souvent la joie de rencontrer, à la tête des collections qu'il étudiait, des savants de son propre type, prompts à mettre à la disposition de leurs confrères les richesses dont ils avaient la garde. Là était pour notre ami l'une des joies profondes de ces recherches: la possibilité de les communiquer. Rien ne le touchait plus que cette bonne volonté, cette communion d'esprit; rien ne le réjouissait autant que de se voir abordé par un questionneur, sollicité de donner son avis, d'ouvrir ses dossiers. Sa libéralité était sans bornes. Tout ce qu'il avait accumulé, fiches ou livres, était offert à tous ceux qui cherchaient réponse à un problème.

Car cet homme, qui par ses goûts intellectuels se sentait à part de l'homme dans la rue, qui par certains côtés de son caractère était un "singulier», un exemplaire tiré à un numéro unique, était en même temps, nature pleine de contrastes, avide de société et surtout d'amitié. Il aimait le jeu des idées, les visiteurs avec lesquels il pouvait soulever un problème, discuter «de omni re scibili», feuilleter un livre, suivre la marche d'une idée dans le monde. Tout comme, méticuleux et exigeant dans ses travaux d'érudition, il se livrait, dans la conversation, aux paradoxes les plus osés. Gymnastique acrobatique qui lui aérait les idées et le voyait ensuite revenir rajeuni et plus sobre que jamais à ses incunables, à leurs imprimeurs, à leurs illustrateurs, à leurs auteurs de toutes les époques. Là, plus de fantaisie, plus de cavalcades sur des chimères; la rigueur de l'exactitude poussée à la limite du possible.

Toutes les notes, les monographies, les articles, touchant à l'histoire de la médecine, que Klebs fit paraître au cours d'une trentaine d'années, et pour finir ses Incunabula, parus en 1938, n'étaient que des travaux d'approche. En particulier, cette dernière «somme»n'est qu'un point de départ: comment se rendre compte de ce qu'était la médecine au 15 siècle si l'on ne commençait par en établir l'exacte bibliographie? Jusqu'à ce que ce tableau préliminaire fût au point, on ne s'en ferait jamais qu'une idée plus ou moins vague, attribuant à telle œuvre une 
valeur excessive, négligeant telle autre, conduit au hasard par ses goûts ou des rencontres occasionnelles. Les Incunabula en main, et ayant en tête le fruit des longues recherches dont ils étaient l'aboutissement, il devenait possible pour Klebs d'animer ce squelette volontairement dépouillé, de le doter de ses muscles, de lui rendre cerveau et organes des sens; la statue allait pouvoir être modelée, et, pour finir, prendre vie.

Personne n'y fût parvenu mieux que lui. Non seulement il avait à la fois la connaissance des détails et la vue de l'ensemble; à son savoir méticuleux et aux idées générales longuement méditées s'ajoutait le complément d'un goût artistique sûr et l'agrément littéraire qui caractérise ses écrits. Le style pédant et morne n'est point son fait. S'il avance avec rigueur dans ses déductions, c'est en parsemant sa route de remarques originales, en surprenant le lecteur par des formules frappées à son coin personnel. Une histoire des idées médicales au $15^{\mathrm{e}}$ siècle, sortie de sa plume, eût été un rare régal pour les esprits sensibles à la beauté comme au savoir. Il eut un temps l'espoir de la mener à bien. Lorsqu'il publia les Incunabula, il avait soixante-huit ans. Sa santé commençait à fléchir, mais l'esprit était en pleine activité. Quelques années encore, et la construction eût pu être achevée. Le sort ne l'a pas permis. Il eût pour cela, avant tout, fallu l'aide indispensable pour les innombrables vérifications de détail qui doivent accompagner la rédaction d'une pareille œuvre d'ensemble, assurer jusqu'au moindre moëllon de l'édifice. Aide difficile à trouver; un essai, qui semblait prometteur, ne réussit pas. L'an d'après, la guerre reprit et s'étendit de plus en plus, à la souffance de l'homme sensible qui comptait des amis dans tous les pays civilisés. Ses vieux amis des Etats-Unis, ceux qui étaient depuis quarante ans les plus proches de son cœur, au courant de toutes ses aspirations, lui furent enlevés par la mort. Par dessus tout, Madame Klebs, son bon génie, la grâce et la paix des Terrasses, frappée d'apoplexies successives, n'était plus que l'ombre d'elle-même, une ombre qui vivait déjà hors du triste royaume des vivants. La possibilité de travailler, le goût même du travail, s'effritèrent ainsi en quelques mois chez notre ami. De plus en plus, ses pensées restèrent centrées sur la couche de la malade. Il l'entourait incessamment des attentions les plus touchantes, avec l'espoir de voir quelque étincelle de vie consciente se ranimer par ses soins, croyant même parfois y être parvenu pour un instant. Mais le grand projet fut abandonné. Il fallut renoncer à donner vie au squelette monté avec amour. 
«Pendet opus interruptum». Ainsi la vraie œuvre d'Arnold Klebs consiste moins, en définitive, dans les écrits qu'il laisse que dans sa personnalité même. Sans doute, on ne prend pas l'un d'eux en main sans y trouver de quoi enrichir son savoir ou donner à sa fantaisie l'occasion de se mettre en campagne. Qu'il parle de l'inoculation - où il se plaira à décocher quelques flèches à la vaccination — ou de la balnéologie au moyen-âge; de la peste ou de ses chers Herbiers du quinzième siècle; des manuscrits de Léonard de Vinci ou de la paléopathologie dont il voudrait voir le domaine mieux défini; ou des incunables et du mouvement intellectuel dont ils sont d'abord les témoins pour en devenir bientôt le ferment irrésistible, — partout il laisse sa marque. Il l'eût fait mieux encore s'il lui avait été donné de compléter sa liste d'incunables par le tableau, à la fois pittoresque, raisonné et philosophique de la pensée médicale au quinzième siècle. Mais mieux que personne il savait que si les écrits seuls assurent à un nom une certaine survie, ils portent en eux le germe de déchéance et de mort qui est le lot de l'homme. A peine un ouvrage est-il paru, que ses lacunes, ses défauts, ses erreurs, ressortent, marque de l'inévitable tare originelle, et font de lui en peu d'années, un objet de curiosité plus qu'une source d'inspiration. Lui-même, pour son Inoculation, avait dressé une bibliographie qu'il croyait assez complète, pour constater bien vite qu'il y avait lieu à la doubler. De quoi il ne songeait pas à se plaindre. Chaque génération n'a-t-elle pas pour mission, en reprenant la route parcourue par les anciens, d'avancer au delà du point atteint? Il est nécessaire et bon que les après-venant fassent mieux que nous; il doit nous suffire d'avoir fait ce que nous pouvions, mieux que nos prédécesseurs.

Arnold Klebs n'était pas un caractère simple - à supposer qu'il en existe. Il était, avec sa lucidité et sa franchise, le premier à s'en rendre compte, et à en souffrir; d'autant plus qu'il y retrouvait un trait de son père: «Der Geist der stets verneint ... C'est un ictus en nous, auquel on ne peut résister; souvent embarrassant et ennuyeux pour les autres, malheureusement", m'écrivait-il un jour (1 ${ }^{\text {er }}$ avril 1940). Sa carrière n'a pas été unie; quinze ans d'activité médicale ont suffi à le détacher de la profession malgré les succès et les honneurs qu'elle lui avait procurés. Mais alors déjà, et plus encore dans le champ nouveau où se déploiera désormais son activité, si l'objet de celle-ci a changé, un trait fondamental de notre ami s'est toujours manifesté: se mettre au service d'autrui. Ses aspérités de surface, qu'il n'arrivait pas toujours à adoucir 
au bon moment, les orages qui pouvaient éclater à l'improviste, le sentiment qu'il avait de ne pouvoir travailler que dans l'isolement, cet isolement dont il n'a, pour finir, que trop éprouvé l'amertume; tout cela faisait bon ménage en lui avec le passionné désir de se rendre utile. Ses livres, son argent, ses idées, le fruit de ses recherches, tout cela lui paraissait peu de chose s'il ne pouvait le mettre à votre disposition. Aucune peine ne le rebutait lorsqu'il s'agissait d'une démarche à faire, lui fût-elle même désagréable et le solliciteur parfaitement inconnu de lui. Voir arriver un collègue, l'accueillir, le choyer, l'aider dans ses recherches, discuter avec lui de tout ce qui peut toucher à la marche de l'esprit humain, voilà ce qu'il aimait par dessus tout. Il en oubliait pour un moment son pessimisme foncier, ${ }^{1}$ et après avoir jugé sévèrement l'homme en général et lui-même en particulier, laissait la joie de servir prendre le dessus et lui dicter les gestes les plus délicats. Il possédait le génie de l'amitié, ou plutôt, il en était possédé. C'est, il le confesse, cela qui le séduisit à son premier contact avec une école américaine, où il voyait, comme jamais auparavant, la collaboration des maîtres et des élèves, unis comme une famille.

C'est cet esprit de bon accord, de poursuite en commun d'un même idéal, en parfaite indépendance d'esprit mais dans l'affection réciproque, qu'il eût voulu pouvoir propager chez nous, une fois rentré en Suisse. Aux Etats-Unis, il avait été l'un des fondateurs de la grande Association nationale contre la tuberculose; en Suisse, c'est avant tout à lui que la Société d'histoire de la médecine et des sciences naturelles doit sa naissance. Il la voyait, en esprit, débordante de vie, d'entrain, de jeunesse, recruter par milliers tous ceux qu'intéresse l'histoire de l'esprit humain, leur faire mettre en commun leurs recherches, leurs espoirs, leurs désillusions aussi; procurer à tous ses membres le sentiment qu'ils étaient compris et appuyés par leurs confrères... Une abbaye de Thélème modernisée. La réalité ne répondit que de loin à ce tableau idéal. Chacun a trop d'obligations, et quand ce ne serait que celle de gagner sa vie, pour se libérer comme cela eût été nécessaire; l’idéal entrevu était trop séduisant pour n'être pas chimérique. Klebs souffrit de constater le médiocre écho qu'éveilla son appel. La Société compte des personnalités

1 «Ce qui m'intéresse maintenant plus que toute autre chose, c'est la pensée humaine, ou peut-être sa presque totale absence dans la formation du destin humain», lettre du ler avril 1940. 
de haute valeur, mais ressemble à beaucoup de ses sœurs; entre les réunions annuelles, chacun vit et travaille pour soi; ce ne sont pas des milliers de membres jeunes et pleins d'allant qu'elle groupe, mais une centaine de messieurs réfléchis et posés. Notre pays a ses us et coutumes; le sang qui circule dans nos artères est un vieux sang qui ne change pas aisément ses caractères.

Du moins notre ami a-t-il fait ce qui dépendait de lui pour lui infuser le rajeunissement qui lui paraissait désirable. Après lui, sa bibliothèque continuera, mais aux Etats-Unis, à représenter sa volonté de recherche consciencieuse, étant destinée à rejoindre celle de son ami Harvey Cushing à l'Université de Yale. Bientôt il ne restera que peu de signes tangibles de sa présence dans notre pays. A nous de cultiver avec d'autant plus de soin le souvenir de son exemple: se consacrer à la recherche du vrai, se dévouer, se donner, être sincère sans défaillance. Une lettre de lui se terminait par une citation prise de l'un des derniers billets de son père - avec lequel ses rapports n'avaient pas toujours été faciles - : "on peut différer d'avis, changer d'opinion au cours du temps; l'amour reste». Testament spirituel auquel à son tour le fils a fait honneur.

Sur la porte du Cottage, donnant accès à la pièce où il a tant travaillé et où, pour finir, il est mort, est inscrite, en lettres rétrogrades, la maxime de Léonard: «Se tu sarai solo, tu sarai tutto tuo». - Seul, il l'a beaucoup été, et il n'en pouvait être autrement; seul il est mort, sans même que sa femme, dans la chambre au-dessus, s'en rendît compte. Mais s'il a accepté son isolement pour sauvegarder son indépendance d'esprit et n'obéir qu'aux injonctions de sa conscience, il l'a corrigé par sa générosité à faire participer chacun à ses trésors. Ce n'était pas pour les caresser en avare qu'il réunissait documents et livres, mais au contraire pour les communiquer le plus libéralement possible. Si bien que sa conduite fournissait à Léonard le complément le plus explicite :

Sois pleinement toi, et pour cela donne toi.

Le Mont, mars 1943.

E. Olivier.

La liste des publications d'Arnold-C. Klebs se trouve dans le Bulletin of the History of Medicine, Baltimore, VIII/3, 1940, p. 523-532; numéro spécial consacré à Klebs à l'occasion de ses soixante-dix ans. 